\title{
GHB ROLLER-COASTER: SEROTONIN SYNDROME AND WITHDRAWAL
}

\author{
Dina Bosnjak Kuharic, Jaksa Vukojevic, Nikola Zaja, Goran Arbanas \& Antonia Puljic \\ University Psychiatric Hospital Vrapce, Zagreb, Croatia
}

received: 5.6.2019;

revised: 11.7.2019;

accepted: 27.8.2019

$* * * * *$

\section{INTRODUCTION}

Over the past decade, recreational drug gammahydroxybutyrate (GHB) drew the attention of clinicians worldwide due to its puzzling effects and a variety of clinical presentations connected with GHB-intoxication (Kamal et al. 2017). Despite the substantial increase in published case reports (Drasbek et al. 2006, Gonzalez \& Nutt 2005, Zvosec et al. 2011), when compared to other club drugs, the prevalence of its use is still relatively low, while the complications of intoxication, withdrawal syndrome and dependence are disproportionately high, usually requiring prolonged and intensive treatment (Dijkstra et al. 2017) and potentially leading to a fatal outcome (Corkery et al. 2016). Several risk factors, including a history of polydrug dependence (Caputo et al. 2009), sleep disorders (Stein et al. 2011) and psychiatric disorders, especially anxiety, depression and borderline personality disorder (Corkery et al. 2016, Dijkstra et al. 2017, Bornovalova et al. 2005) have been identified for developing GHB dependence (Kamal et al. 2017). Additionally, the anxiolytic and antidepressant properties of GHB itself could contribute to its high abuse potential (Kamal et al. 2017).

One of the most severe adverse effects of almost all antidepressants is serotonin syndrome, a condition characterized by a triad of altered mental status, autonomic dysfunction and neuromuscular abnormalities (Martin 1996). Laboratory findings may include liver impairment, renal impairment, leukocytosis and lower bicarbonate levels (Volpi-Abadie et al. 2013). As the diagnosis of serotonin syndrome is based only on clinical observation and exclusion of other medical conditions, the recognition of this potentially life-threatening condition that requires immediate medical care can be challenging, especially in emergency situations (Martin 1996). Serotonin syndrome is caused by excessive stimulation of serotonin receptors by high serotonin levels through direct serotonin receptor agonism, decreased serotonin breakdown, decreased reuptake of serotonin, increased release of serotonin or increased synthesis of serotonin (Bartlett 2017). Apart from antidepressants, other medications that have serotonin agonist activity, e.g., analgesics, antibiotics, antimigraine medications, herbal supplements, and over-thecounter drugs can lead to the syndrome. When it comes to illicit drugs, serotonin syndrome is usually associated with amphetamine, methylenedioxymethamphetamine (MDMA) and cocaine (Bartlett 2017).

Here, we present the first case (to our knowledge) of serotonin syndrome following intoxication of GHB. Our aim was to describe this specific clinical presentation and investigate possible mechanisms leading to it.

\section{CASE REPORT}

In May 2017, a 39-year-old man was admitted to an intensive care unit conscious, but non-responsive, with hyperpyrexia, diaphoresis, tachycardia, hypertension, and bowel hypermotility. His whole body was in extremely high tonus with clonus, tremor, and hyperreflexia with nystagmus, mydriasis, pupils without proper reaction to light. Additional information was obtained from his mother who stated that the patient's condition deteriorated rapidly after taking a high dose of "droplets of happiness," which is a name he used for GHB. He started to shiver and hallucinate, became agitated and uncooperative, so his mother called medical emergency service. According to his past medical history, he was in an outpatient psychiatric care due to GHB-dependence and occasional cannabis and ecstasy abuse, with insomnia and symptoms of depression mentioned as the main reasons for his frequent GHB-relapses. He was previously treated with $30 \mathrm{mg}$ of mirtazapine, $900 \mathrm{mg}$ of valproate and $15 \mathrm{mg}$ of diazepam. The toxicology report was positive for GHB, nicotine, caffeine, benzodiazepines, lidocaine, mirtazapine and traces of valproic acid. Laboratory tests showed leukocytosis, signs of liver impairment, electrolyte disturbance and rhabdomyolysis with signs of mild renal insufficiency (Table 1). Serotonin syndrome was suspected and continuous intravenous treatment with high doses of diazepam was started. During the next couple of days, he was unconscious, not reacting to stimuli, in muscular hypertonus with involuntary movements of his left hand and leg. By the fourth day of treatment, a total dose of $710 \mathrm{mg}$ of diazepam was administered intravenously. His symptoms changed from those of serotonin syndrome to those of severe GHB withdrawal with psychotic symptoms. He was agitated, restless, disoriented, inadequate in communication, verbalizing delusions and hallucinations. A consultant psychiatrist recommended $15 \mathrm{mg}$ 
Table 1. Relevant laboratory findings

\begin{tabular}{lcc}
\hline Abnormal laboratory results Day 1 & Value & Reference range \\
\hline Leukocytes & $11.2110^{9} / \mathrm{L}$ & $3.4-9.710^{9} / \mathrm{L}$ \\
Aspartate-aminotransferase & $115 \mathrm{U} / \mathrm{L}$ & $11-34 \mathrm{U} / \mathrm{L}$ \\
Lactate-dehydrogenase & $361 \mathrm{U} / \mathrm{L}$ & $<241 \mathrm{U} / \mathrm{L}$ \\
Creatine-kinase & $3208 \mathrm{U} / \mathrm{L}$ & $<177 \mathrm{U} / \mathrm{L}$ \\
Myoglobin & $2385 \mathrm{U} / \mathrm{L}$ & $19-92 \mu \mathrm{g} / \mathrm{L}$ \\
Creatinine & $124 \mu \mathrm{mol} / \mathrm{L}$ & $64-104 \mu \mathrm{mol} / \mathrm{L}$ \\
CKD-EPI GFR & $64 \mathrm{ml} / \mathrm{min}$ & $90-120 \mathrm{ml} / \mathrm{min}$ \\
Potassium & $2.9 \mathrm{mmol} / \mathrm{L}$ & $3.9-5.1 \mathrm{mmol} / \mathrm{L}$ \\
Calcium (ionized) & $1.10 \mathrm{mmol} / \mathrm{L}$ & $1.18-1.32 \mathrm{mmol} / \mathrm{L}$ \\
\hline
\end{tabular}

Abbreviation: CKD-EPI GFR= The Chronic Kidney Disease Epidemiology Collaboration glomerular filtration rate

of haloperidol daily and switching to peroral diazepam in a dose of $30 \mathrm{mg}$ daily. On the eighth day of treatment, he was fully conscious and in a stable physical condition. Although he could not recall all the details preceding intoxication, he stated that he mistakenly drank a large sip of undiluted GHB after taking his usual diluted dose. During the past months, his daily dose was up to two bottles of GHB (one bottle containing $20 \mathrm{ml}$ ), diluted with juice and taken in divided doses about 15 times a day. He verbalized motivation for treatment of GHB-dependence and was transferred to a psychiatric unit.

\section{DISCUSSION}

Having a versatile pharmacological profile with direct or indirect effects on four major neurotransmitters (Kamal et al. 2016), GHB can lead to various clinical presentations and show intricate interactions with other medications. GHB has been shown to have a positive effect on serotonin (Kamal et al. 2017, Gobaille et al. 2002), whether by increasing tryptophan reuptake and potentiating serotonin turnover (Gobaille et al. 2002, Hedner \& Lundborg 1983), or whether by indirectly acting through GABAB and dopamine activity mediated via the serotonergic dorsal raphe nucleus (Burman et al. 2003, Ferré \& Artigas 1993). In the case of our patient who reported previous excessive use of GHB, intoxication with high doses of GHB could have increased serotonin levels and cause adverse effects in the form of serotonin syndrome followed by a severe GHB-withdrawal syndrome. Moreover, the uncommon way of GHB consummation as a concentrated liquid prior to the development of symptoms could also influenced this specific presentation.

When it comes to other mechanisms that could lead to the development of serotonin syndrome in this particular case, we have considered the use and/or abuse of mirtazapine as a potential contributor. According to previous literature, different antidepressants can increase the risk for serotonin syndrome, and GHB users seem to be prone to abuse different illicit drugs and medications to relieve their symptoms (Kamal et al. 2017). As the main reasons for using "droplets of happiness" and other illicit drugs (combined or separately) were depression and insomnia, in the case of our patient we cannot rule out the possibility of antidepressant misuse as a part of his self-treatment as well. However, while there are some reports connecting mirtazapine with serotonin syndrome (Ubogu \& Katirji 2003, Hernández et al. 2002), this theory has many ambiguities and is still not widely accepted (Isbister \& Whyte 2003). We cannot be sure that mirtazapine had influence on this presentation, nor alone nor through a possible interaction with GHB.

Additionally, problems with diagnosing serotonin syndrome could also contribute to the complexity of our case. In other words, serotonin syndrome is considered to be an under-diagnosed condition as its diagnosis usually relies only on clinical presentation and exclusion of other possibilities, without specific diagnostic methods' results (Martin 1996). Therefore, it is possible that other drug-induced syndromes can mimic serotonin syndrome, and especially in cases like this, with multidrug use and abuse, these other syndromes should be taken into consideration. Anticholinergic poisoning, malignant hyperthermia, neuroleptic malignant syndrome and sympathomimetic poisoning were differential diagnosis possibilities in this case (Martin 1996, Volpi-Abadie et al. 2013), but each of these was excluded due to characteristic symptoms present in our case. Rapid development of symptoms including altered mental status (from confusion and agitation to loss of consciousness), neuromuscular (e.g. tremor, hyperreflexia, high tonus and clonus) and autonomic symptoms (e.g. mydriasis, tachycardia, tachypnea, diaphoresis), and abnormal laboratory tests results (Table 1) led to the diagnosis of serotonin syndrome in our case.

This case is unique as it is the first to describe the development of serotonin syndrome after GHB-intoxication. Considering the polydrug misuse and the comorbid psychiatric conditions in GHB users, in addition to the rising prevalence of GHB use, one must bare in mind the array of possible pharmacological interactions and clinical presentations that can be seen in both GHB intoxication and withdrawal. Although GHB-intoxication could lead to a variety of severe complications, GHB is still not a part of the routine toxicology testing. This, in addition to the observation that serotonin syn- 
drome is frequently unrecognized condition, can result in a prolongation of time for reaching the right diagnosis and starting the adequate treatment in emergency cases where the time is usually lacking. Therefore, when GHB-intoxication is suspected, clinicians should be aware of all possible life-threatening differential diagnosis, including serotonin syndrome, to provide adequate and immediate care and to avoid possible fatal outcome.

\section{Acknowledgements:}

Source of Funding

This research did not receive any specific grant from funding agencies in the public, commercial, or not-forprofit sectors.

\section{Conflict of interest: None to declare.}

\section{Contribution of individual authors:}

Dina Bosnjak Kuharic, Nikola Zaja \& Antonia Puljic collected and interpreted the data and medical information about the presented case.

Jaksa Vukojevic \& Goran Arbanas conducted literature searches and provided summaries of previous literature.

Dina Bosnjak Kuharic \& Jaksa Vukojevic wrote the first draft of the manuscript.

All authors had part in drafting of the manuscript and accepted the final version.

\section{References}

1. Bartlett D: Drug-Induced Serotonin Syndrome. Crit Care Nurse 2017; 37:49-54

2. Bornovalova MA, Lejuez CW, Daughters SB, Zachary Rosenthal $M$ \& Lynch TR: Impulsivity as a common process across borderline personality and substance use disorders. Clin Psychol Rev 2005; 25:790-812

3. Burman KJ, Ige AO, White JH, Marshall FH, Pangalos $M N$, Emson PC et al.: GABA B receptor subunits, $R 1$ and $R 2$, in brainstem catecholamine and serotonin neurons. Brain Res 2003; 970:35-46

4. Caputo F, Francini S, Stoppo M, Lorenzini F, Vignoli $T$, Del Re A et al.: Incidence of craving for and abuse of gamma hydroxybutyric acid (GHB) in different populations of treated alcoholics: an open comparative study. J Psychopharmacol 2009; 23:883-90

5. Corkery JM, Loi B, Claridge H, Goodair C, Corazza O, Elliott $S$ et al.: Gamma hydroxybutyrate (GHB), gamma butyrolactone $(G B L)$ and 1,4-butanediol $(1,4-, B D ; B D O)$ :
6. A literature review with a focus on UK fatalities related to non-medical use. Neurosci Biobehav Rev 2015; 53:52-78

7. Dijkstra BA, Kamal R, van Noorden MS, de Haan H, Loonen AJ \& De Jong CA: Detoxification with titration and tapering in gamma-hydroxybutyrate (GHB) dependent patients: The Dutch GHB monitor project. Drug Alcohol Depend 2017; 1:164-173

8. Drasbek K, Christensen J, Jensen K: Gamma-hydroxybutyrate- a drug of abuse. Acta Neurol Scand 2006; 114:145-56

9. Ferré $S$ \& Artigas F: Dopamine D 2 receptor mediated regulation of serotonin extracellular concentration in the dorsal raphe nucleus of freely moving rats. $J$ Neurochem 1993; 61:772-775

10. Gobaille S, Schleef $C$, Hechler V, Viry S, Aunis D \& Maitre M: Gamma-hydroxybutyrate increases tryptophan availability and potentiates serotonin turnover in rat brain. Life Sci 2002; 70:2101-2112

11. Gonzalez A \& Nutt DJ: Gamma hydroxy butyrate abuse and dependency. J Psychopharmacol 2005; 19:195-204

12. Hedner $T \&$ Lundborg P: Effect of gamma-hydroxybutyric acid on serotonin synthesis, concentration and metabolism in the developing rat brain. J Neural Transm 1983; 57:39-48

13. Hernández JL, Ramos FJ, Infante J, Rebollo $M$ \& González-Macias J: Severe serotonin syndrome induced by mirtazapine monotherapy. Ann Pharmacother 2002; 36:641-3

14. Isbister GK \& Whyte IM: Adverse reactions to mirtazapine are unlikely to be serotonin toxicity. Clin Neuropharmacol 2003; 26:287-8; author reply 289-90

15. Kamal RM, van Noorden MS, Wannet W, Beurmanjer H, Dijkstra BA \& Schellekens A: Pharmacological Treatment in $\gamma$-Hydroxybutyrate $(G H B)$ and $\gamma$-Butyrolactone $(G B L)$ Dependence: Detoxification and Relapse Prevention. CNS Drugs 2017; 31:51-64

16. Kamal RM, van Noorden MS, Franzek E, Dijkstra BA, Loonen AJ \& De Jong CA: The Neurobiological Mechanisms of Gamma-Hydroxybutyrate Dependence and Withdrawal and Their Clinical Relevance: A Review. Neuropsychobiology 2016; 73:65-80

17. Martin TG: Serotonin syndrome. Ann Emerg Med 1996; 28:520-6

18. Stein LA, Lebeau R, Clair M, Martin R, Bryant M, Storti S et al.: A web-based study of gamma hydroxybutyrate (GHB): patterns, experiences, and functions of use. Am J Addict 2011; 20:30-9

19. Ubogu EE \& Katirji B: Mirtazapine-induced serotonin syndrome. Clin Neuropharmacol 2003; 26:54-7

20. Volpi-Abadie J, Kaye AM \& Kaye AD: Serotonin syndrome. Ochsner J 2013; 13:533-40

21. Zvosec DL, Smith SW, Porrata T, Strobl AQ \& Dyer JE: Case series of 226 gamma-hydroxybutyrate-associated deaths: lethal toxicity and trauma. Am J Emerg Med 2011; 29:319-32

Correspondence:

Dina Bosnjak Kuharic, MD, PhD

University Psychiatric Hospital Vrapce, Department for diagnostics and intensive care

Bolnicka cesta 32, 10090 Zagreb, Croatia

E-mail:dina.bosnjak@gmail.com 